\title{
Quantitative Study of Macroscopic Quantum Tunneling in a de SQUID: A System with Two Degrees of Freedom
}

\author{
Shao-Xiong Li, Yang Yu, Yu Zhang, Wei Qiu, and Siyuan Han \\ Department of Physics and Astronomy, University of Kansas, Lawrence, Kansas 66045 \\ Zhen Wang \\ KARC, Communication Research Laboratory, Ministry of Posts and Telecommunications, \\ 588-2 Iwaoka, Owaoka-cho, Nishi-ku, Kobe 651-24, Japan
}

(Received 2 October 2001; published 7 August 2002)

\begin{abstract}
To test whether the theory of macroscopic quantum tunneling (MQT) is applicable to systems with 2 degrees of freedom, we experimentally investigated the switching current distribution of a dc SQUID. Using sample parameters determined from measurements at $T=4.2 \mathrm{~K}$, we are able to make quantitative comparison to the theories from $8 \mathrm{mK}$ to $4.2 \mathrm{~K}$. The excellent agreement between the data and the MQT theory demonstrates that tunneling from the zero-voltage state of the dc SQUID is well described by the quantum mechanics.
\end{abstract}

DOI: 10.1103/PhysRevLett.89.098301

Whether quantum mechanics is valid for macroscopic variables is one of the most fascinating issues of fundamental physics [1]. The experimental studies, especially quantitative tests of the theory for macroscopic variables in the quantum regime, provide important insights to our understanding of the physical world. In the past few years, devices based on the Josephson effect, such as the Josephson junction (JJ) and the superconducting quantum interference device (SQUID), have been proven effective systems to perform this test. For instance, in a JJ, the phase difference $\delta$ across the junction is a macroscopic variable and the dynamics of the junction is identical to a particle's motion in a one-dimensional (1D) washboard potential. Experiments in 1D systems, such as current biased JJs and rf SQUIDs, have yielded results in very good agreement with the theoretical predictions of macroscopic quantum tunneling (MQT) [2-6]. In contrast, experiments in systems with 2 degrees of freedom (2DF) have produced significant divergences. For example, using a dc SQUID, which contains two JJs and, hence, has two macroscopic degrees of freedom, Sharifi, Gavilano, and Harlingen (SGH) reported the observation of anomalous suppression of thermal activation (TA) from the two-dimensional (2D) potential well of a dc SQUID [7]. SGH suggested that the apparent suppression of TA rate may rise from an enhanced potential barrier caused by interaction between the two macroscopic degrees of freedom in the dc SQUID. On the other hand, experiments by Han, Lapointe, and Lukens (HLL) [8] and Lefevre-Seguin, Turlot, Urbina, Esteve, and Devoret (LTUED) [9] showed that, in the thermal regime, the activation energy of the 2D SQUIDs agreed very well with the potential barrier. More importantly, SGH's result in the quantum regime significantly disagreed with the theoretical prediction as the measured width of switching current distribution-at temperature well below the quantum-classical crossover temperature $T_{\mathrm{co}^{-}}$ exceeded the theoretical prediction by more than $40 \%$.
PACS numbers: 85.25.Dq, 03.65.-w, 74.50.+r

Notice that this disagreement could not be accounted for by measurement uncertainties and the barrier enhancement suggested by SGH would actually make the matter much worse. However, the HLL and LTUED's experiments did not address the quantum regime so that the experimental evidence available thus far [7] seems to indicate that, despite its great success in describing 1D systems, MQT theory might not be applicable to even the simplest 2D systems, such as dc SQUIDs with no flux bias. Furthermore, the understanding of MQT in dc SQUIDs is crucial to quantum computing with SQUID qubits, in which dc SQUIDs, as the most sensitive flux detectors available [10], serve as the readout devices. In this Letter, we report the results of a quantitative study of the escape rate of an underdamped dc SQUID, a system with 2DF, in both the TA and quantum tunneling regimes. In contrast to SGH's result, our data are in excellent agreement with the MQT theory, demonstrating that quantum mechanics also applies well to macroscopic systems with 2DF.

A dc SQUID consists of two JJs connected in parallel by a small superconducting loop of inductance $L$ (Fig. 1 inset). The critical current of each junction is $I_{0}$ (assuming identical JJs). The macroscopic variables of this system are phases, $\delta_{1}$ and $\delta_{2}$, across the two junctions, respectively. For a dc SQUID with current bias $I$ and flux bias $\Phi_{e} \equiv$ $f \Phi_{0}$, where $\Phi_{0}$ is the magnetic flux quantum, the dynamics of the system can be treated as a fictitious particle of mass $C$, which is the sum of the two junctions' capacitance, moving in a 2D potential [11] $U\left(\varphi, \varphi_{\mathrm{dc}}\right)=$ $E_{J}\left[-\cos \left(\varphi_{\mathrm{dc}} / 2\right) \cos \varphi-x \varphi+\beta_{T} j^{2} / 4\right]$, where $E_{J}=$ $\hbar I_{0} / e$ is the sum of two junctions' Josephson coupling energy, $\varphi \equiv\left(\delta_{1}+\delta_{2}\right) / 2$ is the average phase difference across the device, $\varphi_{\mathrm{dc}} \equiv \delta_{2}-\delta_{1}, j=\left(\varphi_{\mathrm{dc}}-2 \pi f\right) / \beta_{T}$ is the normalized circulating current, $x \equiv I / 2 I_{0}$, and $\beta_{T} \equiv$ $2 \pi L I_{0} / \Phi_{0}$. Figure 1 shows a 3D plot of the de SQUID potential with zero flux bias $(f=0)$ and a small $\beta_{T}$ value $\left(\beta_{T}=0.3\right)$. As one can see, in the $\varphi$ (longitudinal) 
direction, the potential has a sequence of saddle points and local minima (wells) located at $\varphi_{\mathrm{dc}}=0$, while along the $\varphi_{\mathrm{dc}}$ (transverse) direction, the potential rises sharply as $\left|\varphi_{\mathrm{dc}}\right|$ increases. Hence, a particle initially trapped in a potential well would escape along the longitudinal direction near the vicinity of $\varphi_{\mathrm{dc}}=0$.

At high temperatures $\left(T \gg T_{\text {co }}\right)$, escape from the $2 \mathrm{D}$ potential well is dominated by TA over the potential barrier with the transition rate given by $[11,12]$

$$
\Gamma=\frac{\Omega}{2 \pi} a_{t} \exp \left(-\frac{\Delta U}{k_{B} T}\right),
$$

where $\Delta U$ is the minimum height of the potential barrier (i.e., through the saddle point), $T$ is temperature, and $a_{t}$ $\left(0<a_{t}<1\right)$ is a damping dependent factor [13]. The "attempt frequency" $\Omega$ is given by $\Omega \equiv \omega_{\mathrm{lw}}\left(\omega_{\mathrm{tw}} / \omega_{\mathrm{ts}}\right)$, where $\omega_{\mathrm{lw}}\left(\omega_{\mathrm{tw}}\right)$ is longitudinal (transverse) small oscillation frequency in the well, and $\omega_{\mathrm{ts}}$ is the transverse oscillation frequency at the saddle. Equation (1) is equivalent to the TA rate of a $1 \mathrm{D}$ system having the attempt frequency renormalized by a factor of $\omega_{\mathrm{tw}} / \omega_{\mathrm{ts}}$. For dc SQUIDs with $\beta_{T} \ll 2 \pi$, such as the one studied here and in [7], the ratio $\omega_{\mathrm{tw}} / \omega_{\mathrm{ts}}$ is close to unity. Hence, the TA rate of dc SQUIDs with $\beta_{T} \ll 2 \pi$ should closely follow that of a 1D system.

At $T \ll T_{\text {co }}$, MQT becomes the dominant escape mechanism. The tunneling rate of dc SQUIDs with $f=0$ is given by [14]:

$$
\begin{aligned}
\Gamma & =f_{2 \mathrm{D}}\left(\alpha, \alpha_{c}\right) \frac{\omega_{0}}{2 \pi} \sqrt{120 \pi}\left(7.2 \frac{\Delta U}{\hbar \omega_{0}}\right)^{1 / 2} \exp \left(-7.2 \frac{\Delta U}{\hbar \omega_{0}}\right) \\
& =f_{2 \mathrm{D}}\left(\alpha, \alpha_{c}\right) \Gamma_{1 \mathrm{D}},
\end{aligned}
$$

where $\omega_{0}=\left(2 \pi I_{0} / C_{J} \Phi_{0}\right)^{1 / 2}\left(1-x^{2}\right)^{1 / 4}$ is the plasma frequency and $C_{J}$ is the shunt capacitance of each junction. $f_{2 \mathrm{D}}\left(\alpha, \alpha_{c}\right)$ is a dimensionless function of $\alpha \equiv 2 \sqrt{2} \beta_{T}^{-1} \times$ $(1-x)^{-1 / 2}$ and $\alpha_{c} \equiv-90 \exp \left(-2 \pi T_{\mathrm{co}} / T\right)+5 / 4$, where $\alpha$ is a measure of interaction between the $2 \mathrm{DF}, \alpha_{c}$ is the

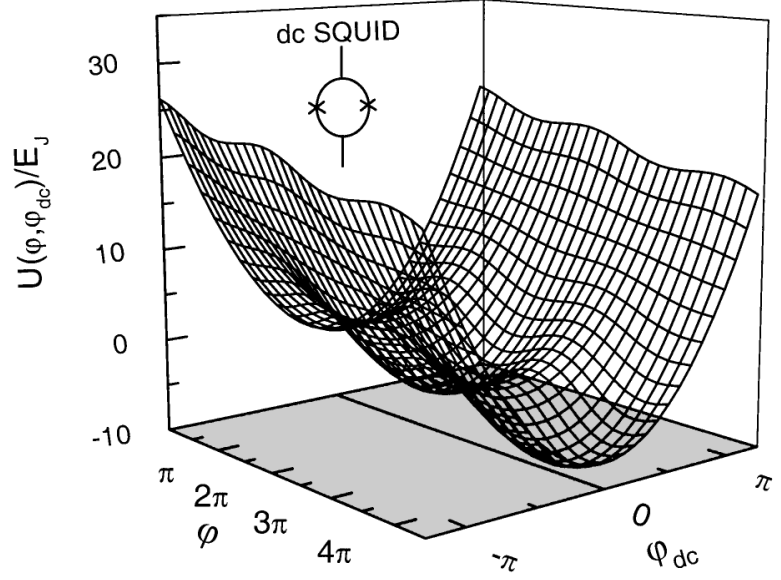

FIG. 1. The 2D potential for a dc SQUID with $f=0, \beta_{T}=$ 0.3 , and $x \equiv I_{b} / 2 I_{0}=0.25$. The inset is a schematic of the dc SQUID. critical parameter for instanton splitting, and $T_{\mathrm{co}}=$ $\hbar \omega_{0} / 2 \pi k_{B}$ is the quantum-thermal crossover temperature. Equation (2) clearly shows that the tunneling rate of a dc SQUID with zero flux is closely related to that of a single Josephson junction. In particular, for de SQUIDs with $\beta_{T} \ll 2 \pi$, such as our sample and that of SGH $\left(\beta_{T} \simeq\right.$ 0.4 ), there is no instanton splitting and $f_{2 \mathrm{D}} \simeq 1$. Notice that the disagreement between the result of [7] and the MQT theory is so significant that no reasonable adjustment of sample parameters could reconcile the data with Eq. (2).

The switching current distribution $P(x)$ is related to the escape rate through

$$
P(x)=\frac{\Gamma(x)}{d x / d t} \exp \left[-\frac{1}{d x / d t} \int_{0}^{x} \Gamma\left(x^{\prime}\right) d x^{\prime}\right] .
$$

To quantitatively compare experimental data with various theories, it is convenient to use the "escape temperature" $T_{\text {esc }}$ defined through [15] $\Gamma=\Omega / 2 \pi \exp \left(-\Delta U / k_{B} T_{\text {esc }}\right)$. It is straightforward to show that, for TA $T_{\text {esc }} \simeq T$, while for quantum tunneling $T_{\mathrm{esc}} \simeq T_{\mathrm{co}}$. For dc SQUIDs biased at $x \leqslant 1, T_{\text {esc }}$ depends linearly on $\sigma^{3 / 2}$ [16], where $\sigma$ is known as the width of $P(x)$. Because in our experiment the widths were extracted directly from the measured $P(x)$, which did not involve the use of any theoretical model and sample parameter, it was used in our data analysis and presentations.

The sample was a dc SQUID consisting of two nominally identical $\mathrm{NbN} / \mathrm{AlN} / \mathrm{NbN}$ JJs each having a diameter of $2 \mu \mathrm{m}$. The critical current of the dc SQUID is $2 I_{0}=$ $35.8 \mu \mathrm{A}$, which was determined by fitting $P(x)$ at $4.2 \mathrm{~K}$, where the system was in the thermal regime. Because of the large energy gap of $\mathrm{NbN}(2 \Delta \simeq 5.4 \mathrm{meV}), I_{0}$ remained constant below $4.2 \mathrm{~K}$. Notice that in the quantum regime it is essential to have an independent measurement of the sample's shunt capacitance for making a quantitative comparison between the data and theoretical predictions. In our experiment, the capacitance of the dc SQUID, $C=2 C_{J}=$ $380 \pm 30 \mathrm{fF}$, was determined from resonant activation (RA) measurement [15] at $4.2 \mathrm{~K}$. The RA data also yielded a quality factor, $Q \equiv R C \omega_{0} \approx 200$, indicating that the effect of damping on tunneling at $T \ll T_{\text {co }}$ was negligible $[17,18]$. The inductance of the dc SQUID loop, estimated from the modulation depth of critical current and loop geometry, is $L=5 \pm 1 \mathrm{pH}$. Using these sample parameters, the crossover temperature is found to be $T_{\mathrm{co}}=0.29 \mathrm{~K}$.

We measured the switching current distribution of the dc SQUID using a time-of-flight technique similar to that described in [19]. Each measured distribution consisted of $2 \times 10^{4} 20000$ escape events. Because heating and external noise could cause the width $\sigma$ to flatten out at low temperatures, which could be mistaken as the evidence for MQT, cautions must be taken to eliminate them. In our experiment, the dc SQUID was enclosed in a helium-filled copper sample cell thermally anchored to the mixing chamber of a dilution refrigerator. EMI filters, cryogenic low-pass filters and microcoax microwave filters [20], 
battery-powered low-noise preamplifiers, and shielded enclosure were used to protect the sample from external noise. Connections to the computer and ac-powered instruments were made via optically coupled isolation amplifiers. Extensive diagnostic tests were made using low critical current $\left(I_{c} \sim 1-10 \mu \mathrm{A}\right)$ junctions to ensure that the effects of noise from the environment and measurement circuitry was negligible down to $8 \mathrm{mK}$. Distributions with $\sigma<15 \mathrm{nA}$ have been observed using the same setup, demonstrating the effectiveness of the shielding. A mumetal cylinder provided $\sim 60 \mathrm{~dB}$ attenuation to shield the sample from ambient magnetic field fluctuations. For improved stability, the data were taken with zero flux bias $(f=0)$ and the temperature was regulated to within $\pm 1 \%$ and $\pm 0.3 \%$ of the set point at $T$ below and above $0.6 \mathrm{~K}$, respectively.

Figure 2 shows a series of measured switching current distributions and their comparisons with the 2D TA and MQT theories. Notice that Eq. (3) is valid if the temporal escape probability at constant bias currents was $P_{\text {esc }}(t)=$ $1-e^{-\Gamma t}$, which was confirmed by the measured $P_{\text {esc }}(t)$ shown in Fig. 2(a). The data in Fig. 2 show that, in both the thermal $\left(T>2 T_{\mathrm{co}}\right)$ and quantum $\left(T>T_{\mathrm{co}} / 2\right)$ regimes, the distributions, including their shape, width, and position, agree very well with the theoretical predictions. Below $0.15 \mathrm{~K}$ the data were essentially the same, as expected for a system with very low damping $[17,18]$. Tunneling rates obtained from the switching current distributions are plotted as a function of barrier height in Fig. 3. Considering that all theoretical calculations were performed using the

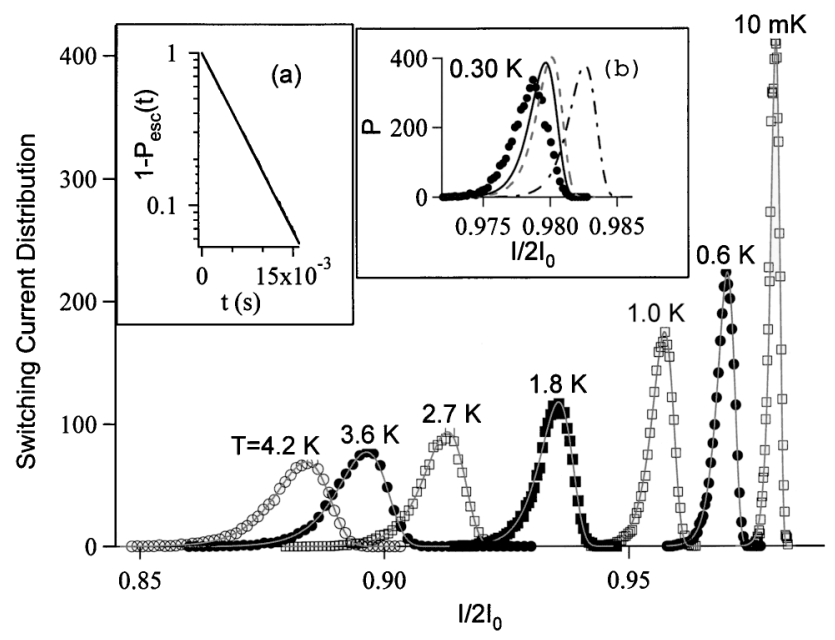

FIG. 2. The measured temperature dependence of the switching current distributions. The symbols are the experimental data and the lines are calculated from the theories of thermal activation and MQT for systems with 2DF. Inset (a): The temporal escape probability at bias $x=0.975$ and $T=10 \mathrm{mK}$ showing the expected exponential decay. Inset (b): Comparison of the measured distribution (solid dots) at $T=300 \mathrm{mK}$ with the theoretical prediction of quantum correction (solid line), $T=0$ MQT (dashed line), and thermal activation (dash-dotted line). sample parameters determined from measurements at $4.2 \mathrm{~K}$, the agreement between the data and theories is quite remarkable. Our data provide strong evidence supporting the validity of MQT theory for systems with 2DF.

However, as shown in Fig. 2(b), in the crossover region the measured distributions deviate significantly from that of $T=0$ MQT theory (the dashed line). By taking into account the effects of thermal enhancement to MQT [17], the agreement between the data and theory (the solid line) improves. However, the remaining disagreement cannot be entirely accounted by the thermally enhanced MQT theory, indicating the need for further studies in this temperature range, where neither TA nor MQT is the dominant escape mechanism.

A critical comparison of the experimental data and various theories can be obtained by examining the $T$ dependence of the distribution width [21]. In Fig. 4 and its inset, the measured $\sigma$ vs $T^{2 / 3}$ is compared with the MQT and TA theories using the sample parameters $2 I_{0}=$ $35.8 \mu \mathrm{A}, C=380 \mathrm{fF}$, and $R=1500 \Omega$. At $T>0.6 \mathrm{~K}$, the data show a linear dependence on $T^{2 / 3}$, as expected from Eq. (1) of TA theory (the solid line) without adjustable parameters. The excellent quantitative agreement between the data and the theory of TA clearly shows that the activation energies were equal to the $2 \mathrm{D}$ potential barriers, in agreement with the results of HLL and LTUED. Below $T=0.15 \mathrm{~K}$, the data became $T$ independent within the uncertainties of measurements. In the quantum limit, the measured $\sigma_{\text {data }}(T \leq 0.15 \mathrm{~K})=40.6 \pm 1.6 \mathrm{nA}$ is MQPsistent with the $41.2 \mathrm{nA}$ calculated from the $T=0$

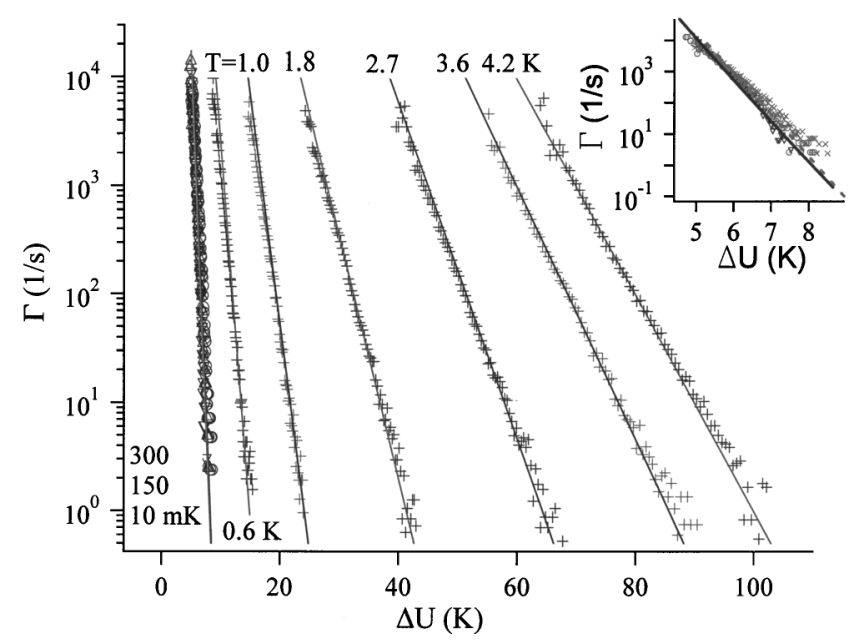

FIG. 3. Tunneling rates vs potential barrier at various temperatures. The symbols are data and the solid lines are theoretical predictions. The first three data sets from the left are $T=10 \mathrm{mK}$ (triangles), $150 \mathrm{mK}$ (circles), and $300 \mathrm{mK}$ (crosses). Inset: The measured (symbols) and predicted (lines) tunneling rates vs barrier height for $T=10 \mathrm{mK}$ (triangles and solid line), $150 \mathrm{mK}$ (circles and dotted line), and $300 \mathrm{mK}$ (crosses and dashed line). 


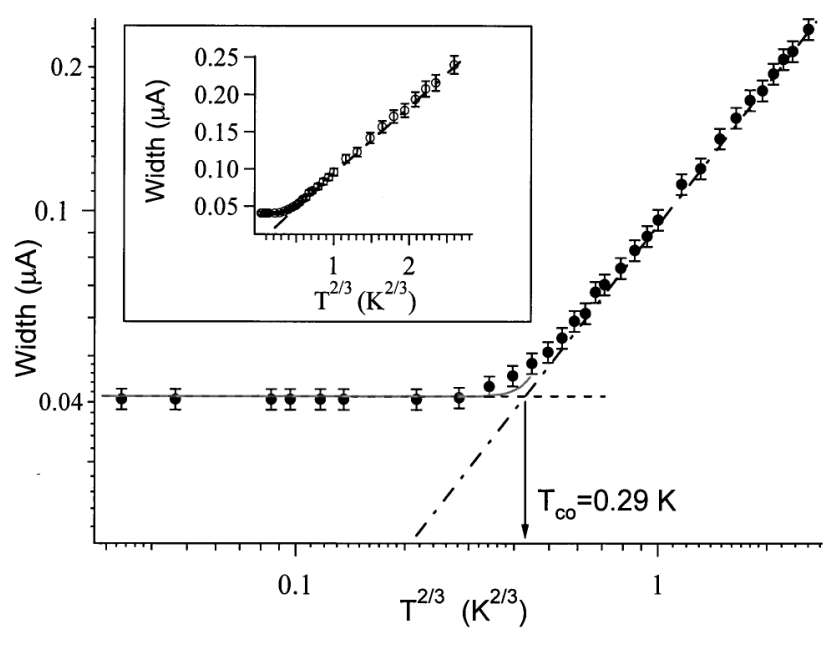

FIG. 4. $\sigma$ vs $T^{2 / 3}$ of the data (symbols) and the predictions of thermally enhanced quantum tunneling of Ref. [12] (solid line), thermal activation (dot-dashed line), and 2D MQT (dashed line) with arrow indicating $T_{\text {co }}$. The log-log plot magnifies the low temperature region, while the inset shows the linear dependence of $\sigma$ on $T^{2 / 3}$ in the thermal regime.

limit, for samples with $Q \gg 1, \sigma$ is almost independent of $C$ but quite sensitive to $R$. The situation is just the opposite in the quantum regime. For instance, using $C=395 \mathrm{fF}$, we found $\sigma=40.6 \mathrm{nA}=\sigma_{\text {data }}(T \leq 0.15 \mathrm{~K})$, while increasing $R$ from $1.5 \mathrm{k} \Omega$ to $1.5 \mathrm{M} \Omega$ results in a negligible change in the calculated width. Hence, the data at $T<$ $0.15 \mathrm{~K}$ again show that in the quantum regime the MQT theory describes the behavior of a system of $2 \mathrm{DF}$ very well.

Finally, we examine the crossover region. From the interception of the straight lines extrapolated from the data in quantum and thermal limits, we found $T_{\text {co }}=$ $0.29 \pm 0.01 \mathrm{~K}$, which agrees very well with the theoretical value of $0.290 \mathrm{~K}$ calculated from the sample parameters. However, for $0.15 \mathrm{~K}<T<0.6 \mathrm{~K}$, the measured $\sigma_{\text {data }}(T)$ deviates systematically from both the $T$-independent behavior of the MQT theory and the simple $\sigma \propto T^{2 / 3}$ scaling behavior of the TA theory (Fig. 4). Similar smooth crossover behavior was observed previously in Josephson junctions by Martinis et al. [5]. Because in both the thermal and quantum limits the data agree very well with the theoretical predictions, the larger width in the crossover region could not be due to heating or external noise. One mechanism that could result in a larger width is the thermal enhancement of MQT, which produces an improved, albeit still unsatisfactory, agreement with the data for $0.15 \mathrm{~K}<T<$ $T_{\mathrm{co}}$. For $T_{\mathrm{co}}<T<0.6 \mathrm{~K}$, the theory of quantum correction [17] produced negligible improvement. In the crossover region, the shape of $P(x)$ also significantly deviates from that calculated from Eq. (3), which could occur when tunneling from excited levels contributes significantly to the escape process [19].

In summary, we have measured the temperature dependence of the switching current distributions of a dc SQUID from well below $\left(<0.025 T_{\text {co }}\right)$ to well above $\left(\sim 14 T_{\text {co }}\right)$ the quantum-classical crossover temperature. The experimental ability to control and characterize the sample and the theoretical capability to accurately model the system facilitate a quantitative comparison between the theories and experiment. Our result in the thermal regime agrees very well with the works of HLL and LTUED, supporting the TA theory. More importantly, without the use of adjustable parameters, the data in the quantum regime agree excellently with the MQT theory, demonstrating incontrovertibly that the theory of MQT correctly describes the behavior of dc SQUIDs - a macroscopic quantum system with 2DF. The result also assures that the MQT theory can be applied to design the readout circuits made of dc SQUIDs for flux based superconducting quantum logic gates.

We thank R. Alexander for technical support. This work was supported in part by AFOSR (F49620-99-1-0205), the state of Kansas (S99041), and the NSF (DMR-9876874).

[1] Quantum Tunneling in Condensed Media, Modern Problems in Condensed Matter Sciences Vol. 34, edited by Y. Kagan and A. J. Leggett (North-Holland, Amsterdam, 1992).

[2] R.F. Voss and R.A. Webb, Phys. Rev. Lett. 47, 265 (1981).

[3] S. Washburn, R. A. Webb, R.F. Voss, and S. M. Faris, Phys. Rev. Lett. 54, 2712 (1985).

[4] D. B. Schwartz, B. Sen, C. N. Archie, and J. E. Lukens, Phys. Rev. Lett. 55, 1547 (1985).

[5] M.H. Devoret, J. M. Martinis, and J. Clarke, Phys. Rev. Lett. 55, 1908 (1985).

[6] J. Clarke et al., Science 239, 992 (1988).

[7] F. Sharifi, J. L. Gavilano, and D. J. V. Harlingen, Phys. Rev. Lett. 61, 742 (1988).

[8] S. Han, J. Lapointe, and J. Lukens, Phys. Rev. Lett. 63, 1712 (1989).

[9] V. Lefevre-Seguin et al., Phys. Rev. B 46, 5507 (1992).

[10] M. Mück, J. B. Kycia, and J. Clarke, Appl. Phys. Lett. 78, 967 (2001).

[11] C. D. Tesche, J. Low Temp. Phys. 44, 119 (1981).

[12] E. Ben-Jacob et al., J. Appl. Phys. 54, 6533 (1983).

[13] M Büttiker, E. P. Harris, and R. Landauer, Phys. Rev. B 28, 1268 (1983).

[14] B. I. Ivlev and Y. N. Ovchinnikov, Sov. Phys. JETP 66, 378 (1987).

[15] J. M. Martinis, M. H. Devoret, and J. Clarke, Phys. Rev. B 35, 4682 (1987).

[16] J. Kurkijärvi, Phys. Rev. B 6, 832 (1972).

[17] H. Grabert, P. Olschowski, and U. Weiss, Phys. Rev. B 36, 1931 (1987).

[18] A. O. Caldeira and A. J. Leggett, Ann. Phys. (N.Y.) 149, 374 (1983).

[19] S. Han et al., Science 293, 1457 (2001).

[20] A. B. Zorin, Rev. Sci. Instrum. 66, 4296 (1995).

[21] A. Garg, Phys. Rev. B 51, 15592 (1995). 